\title{
interprétation géotechnique des courbes de réponse de l'excitation harmonique d'un pieu.
}

\author{
par \\ A. Davis \\ Chef de division CEBTP \\ et \\ P. Guillermain \\ Ingénieur CEBTP
}

Liste des notations

$A_{C}$

$E_{C}$

$E_{D}^{\prime}$

$E_{\text {max }}^{\prime}$

$E_{\min }^{\prime}$

$E_{\infty}^{\prime}$

$E_{s}^{\prime}$

$\mathrm{F}_{\mathrm{o}}$

$t$

$\mathrm{M}_{p}$

N

$r$

d

$v_{c}$

v

$v_{0}$

$\mathrm{v}_{\mathrm{o}} / \mathrm{F}_{\mathrm{o}}$

$\beta$

$p^{\prime}$

$\rho_{C}$

$\sigma$
Section du pieu

Module d'élasticité du béton

Raideur dynamique de la tête du pieu

Raideur dynamique d'un pieu à base rigide

Raideur dynamique d'un pieu sans base

Raideur dynamique d'un pieu d'une longueur infinie

Raideur statique d'un pieu

Force verticale maximale appliquée à la tête du pieu Longueur du pieu

Masse du pieu

Admittance mécanique d'un pieu libre

Rayon du pieu

Diamètre du pieu

Vitesse des ondes longitudinales dans le béton

Vitesse verticale de la tête d'un pieu

Vitesse verticale maximale de la tête d'un pieu

Admittance mécanique de la tête du pieu

Vitesse de propagation des ondes transversales dans le sol

Masse volumique du sol

Masse volumique du béton

Paramètre d'amortissement
Coefficient de Poisson du sol

$\Delta f$

$\mathrm{m}^{2}$

$\mathrm{N} / \mathrm{m}^{2}$

$\mathrm{N} / \mathrm{m}$

$\mathrm{N} / \mathrm{m}$

$\mathrm{N} / \mathrm{m}$

$\mathrm{N} / \mathrm{m}$

$\mathrm{N} / \mathrm{m}$

N

m

$\mathrm{kg}$

$\mathrm{s} / \mathrm{kg}$

m

$m$

$\mathrm{m} / \mathrm{s}$

$\mathrm{m} / \mathrm{s}$

$\mathrm{m} / \mathrm{s}$

$\mathrm{s} / \mathrm{kg}$

$\mathrm{m} / \mathrm{s}$

$\mathrm{kg} / \mathrm{m}^{3}$

$\mathrm{kg} / \mathrm{m}^{3}$

$\mathrm{m}^{-1}$
Ecart de fréquence entre deux pics de résonance

\section{Introduction}

La méthode d'auscultation des pieux et barrettes par impédance mécanique, mise au point par le C.E.B.T.P., est couramment employée dans le contrôle non-destructif des pieux en béton. Jusqu'à présent, cette méthode fournit des renseignements concernant la qualité du béton employé et les caractéristiques géométriques du pieu.

La méthode et son application au contrôle des pieux en place ont fait l'objet de publications antérieures dans les Annales de II.T.B.T.P. [1] et [2].

Or la méthode de l'impédance mécanique se différencie d'autres méthodes de contrôle non-destructif par le fait que l'excitation du pieu engendre une réponse de la tête qui est fonction non seulement de la qualité du pieu, mais aussi de l'interaction du pieu avec le sol d'ancrage et le sol latéral.

Il est donc possible d'interpréter les courbes de réponse qui en résultent pour obtenir des renseignements géotechniques concernant le sol de fondation et l'interaction du pieu sous charge avec ce sol.

\section{Description de la méthode}

Nous rappelons ici la méthode décrite par PAQUET et BRIARD en 1975 [2].

Une force verticale sinusoïdale d'amplitude constante est appliquée sur la tête du pieu. La vitesse de propagation $v_{c}$ des ondes dans le pieu dues à cette force est donnée par :

$$
v_{c}=\sqrt{E_{c} / \rho c}
$$

On fait varier la fréquence d'excitation $f$ de 20 à $1000 \mathrm{~Hz}$ par exemple. L'admittance mécanique du système pieu-sol 
pour une fréquence donnée est caractérisée par la valeur $\mathrm{v}_{\mathrm{o}} / \mathrm{F}_{\mathrm{d}}, \mathrm{v}_{\mathrm{o}}$ étant la vitesse maximale de déplacement de tête du pieu selon son axe, et pouvant être mesurée par un capteur de vitesse placé sur la tête du pieu.

II est commode de tracer, en fonction de la fréquence f la courbe de $/ \mathrm{V}_{0} / \mathrm{F}_{d}$ (figure 1). Aux très basses fréquences on constate que le pieu se comporte comme un ressort dont la souplesse est proportionnelle à la pente de la courbe à l'origine, pente qui, le plus souvent, peut être extrapolée graphiquement. Quand la fréquence augmente, on observe une succession de résonances et d'antirésonances, c'est-à-dire de maximums ou de minimums de vitesse, séparés par des intervalles de fréquence $\Delta f$.

Le sol latéral joue le rôle d'amortisseur. BRIARD a défini cet amortissement par:

$$
e^{-\sigma l}
$$

avec $\sigma l=\frac{l}{\mathrm{r}} \cdot \frac{\rho^{\prime}}{\rho_{\mathrm{C}}} \cdot \frac{\beta}{\mathrm{v}_{\mathrm{C}}}$

Ce coefficient ol est important car il conditionne les possibilités d'application de la méthode. Si l'affaiblissement de propagation est trop important, l'énergie réfléchie sera trop faible pour être décelée et on ne pourra pas ausculter la partie inférieure du pieu.

al dépend du rapport $\frac{l}{r}$ qui caractérise l'élancement du pieu et du rapport $\frac{\rho^{\prime}}{\rho_{C}} \cdot \frac{\beta^{\prime}}{v_{C}}$ qui dépend surtout des propriétés mécaniques du sol latéral.

La courbe dans sa partie résonante oscille autour de la valeur $1 /\left(\rho_{c} \cdot v_{c} \cdot A_{c}\right)$, appelée admittance caractéristique, qui serait d'ailleurs obtenue théoriquement pour un pieu de longueur infinie.

La figure 1 montre une courbe de réponse idéale pour un pieu cylindrique. Sur cette courbe, l'on peut repérer les valeurs de $\mid V_{0} / F_{d}$ minimale et maximale, soient $Q$ et $P$, ainsi que la moyenne géométrique.

$N=1 /\left(\rho_{c} \cdot v_{c} \cdot A_{C}\right)$

La géométrie de la courbe fournit:

$$
P=N \operatorname{coth}(\sigma l)
$$

et

$$
\mathrm{Q}=\mathrm{N} \text { th }(\sigma l)
$$

d'où

$$
\mathrm{N}=\sqrt{(\mathrm{PQ})}
$$

et

$$
\operatorname{coth}(\sigma l)=\sqrt{\frac{P}{Q}}
$$

\section{La raideur dynamique d'un pieu}

L'ancrage terminal réel d'un pieu peut varier entre l'ancrage parfait et l'ancrage nul. La figure 2 indique les conséquences d'une variation des caractéristiques de l'ancrage sur la réponse du pieu. Pour un ancrage raide, la souplesse $S$ à basse fréquence est faible, et pour un ancrage faible et compressible la souplesse devient forte. Ce comportement à basse fréquence peut être représenté par l'inverse de la souplesse, que l'on peut appeler la raideur dynamique, $E^{\prime}$ du pieu.

$$
E_{D}^{\prime}=1 / S
$$

$\mathrm{E}_{\text {jo }}^{\prime}$ correspond a la pente initiale d'une courbe de tassement de la tête d'un pieu en fonction de la charge appliquée sur la tête. II faut rappeler que cette raideur dynamique concerne non seulement la réaction développée à la base du pieu, mais aussi la résistance offerte par le frottement latéral.

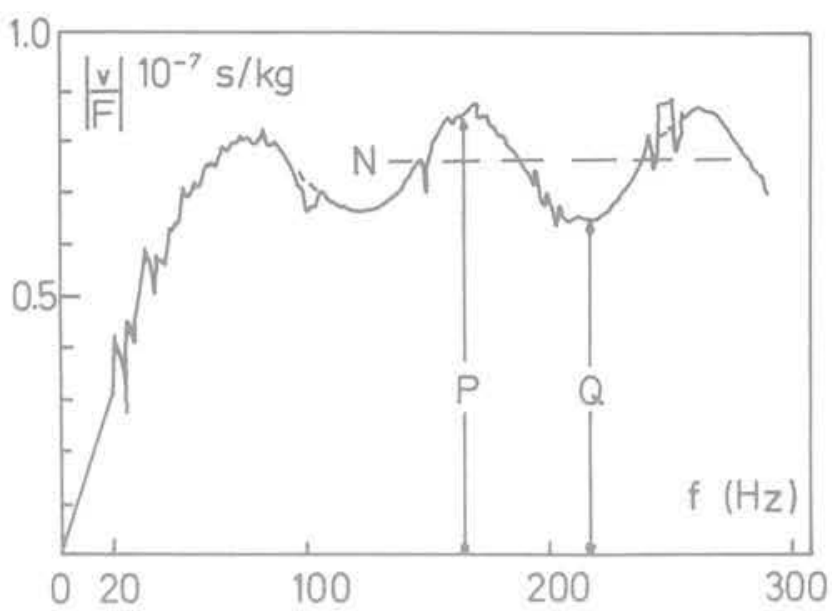

Fig. 1

\subsection{Paramètres contrôlant la raideur dynamique} mesurée à la tête d'un pieu

Afin de comparer les valeurs de $E_{D}^{\prime}$ d'un pieu à un autre, il est nécessaire d'identifier les paramètres qui contrôlent cette raideur.

II s'agit de :

- la longueur du pieu.

- la section du pieu, $A_{c}$

- la forme et l'élancement du pieu.

- la longueur de la partie libre du pieu

- la vitesse de propagation des ondes longitudinales dans le béton, $v_{c}$

- la vitesse de propagation des ondes transversales dans le sol latéral,

- le rapport des modules du sol d'ancrage et du sol latéral (pieu flottant ou pieu travaillant en pointe).

Nous présentons dans le tableau 1 des valeurs de tous ces paramètres pour trois chantiers comparables, deux en France et un en Ecosse. Dans les trois cas, les sols d'ancrage ont sensiblement la même raideur et l'álancement des pieux varie entre $10 / 1$ et $27 / 1$.

A Fos-sur-Mer et à Ambès, les plus gros éléments sont des barrettes de section rectangulaire. La longueur des pieux et des barrettes ne dépasse pas $16 \mathrm{~m}$.

La figure 3 montre la relation entre la raideur dynamique mesurée et la section des pieux pour ces trois chantiers. Les résultats obtenus sont comparables entre eux, chacun des pieux travaillant en pointe.

Depuis l'établissement de cette relation entre $E_{p}^{\prime}$ et $A_{C}$, le CEBTP a eu l'occasion de faire des essais sur plusieurs chantiers situés dans la vallée de la Tamise en Angleterre, aux environs de Londres. Dans tous les cas, les pieux auscultés sont plus longs que ceux de Fos, d'Ambès et de Paisley, avec une longueur variant de 18 à 28 mètres, et un élancement compris entre $20 / 1$ et $50 / 1$. Tous ces pieux traversent des terrains très mous composés de vase, d'argile molle et de tourbe, avant de s'ancrer soit dans des graves de rivière, soit dans la craie.

La figure 4 montre la relation entre $E^{\prime}$ et $A_{c}$ obtenue dans ce cas, ainsi que le fuseau superposé des trois premiers chantiers. On peut noter une croissance quasi-linéaire. Cependant l'augmentation de la longueur des pieux et une épaisse couche d'un sol latéral médiocre ont réduit la raideur pour un pieu de section donnée.

Tous les pieux ayant permis l'établissement de ces courbes sont sans défaut, et la valeur de $v_{c} / 2 \Delta f$ mesurée correspond à la longueur annoncée du pieu. 


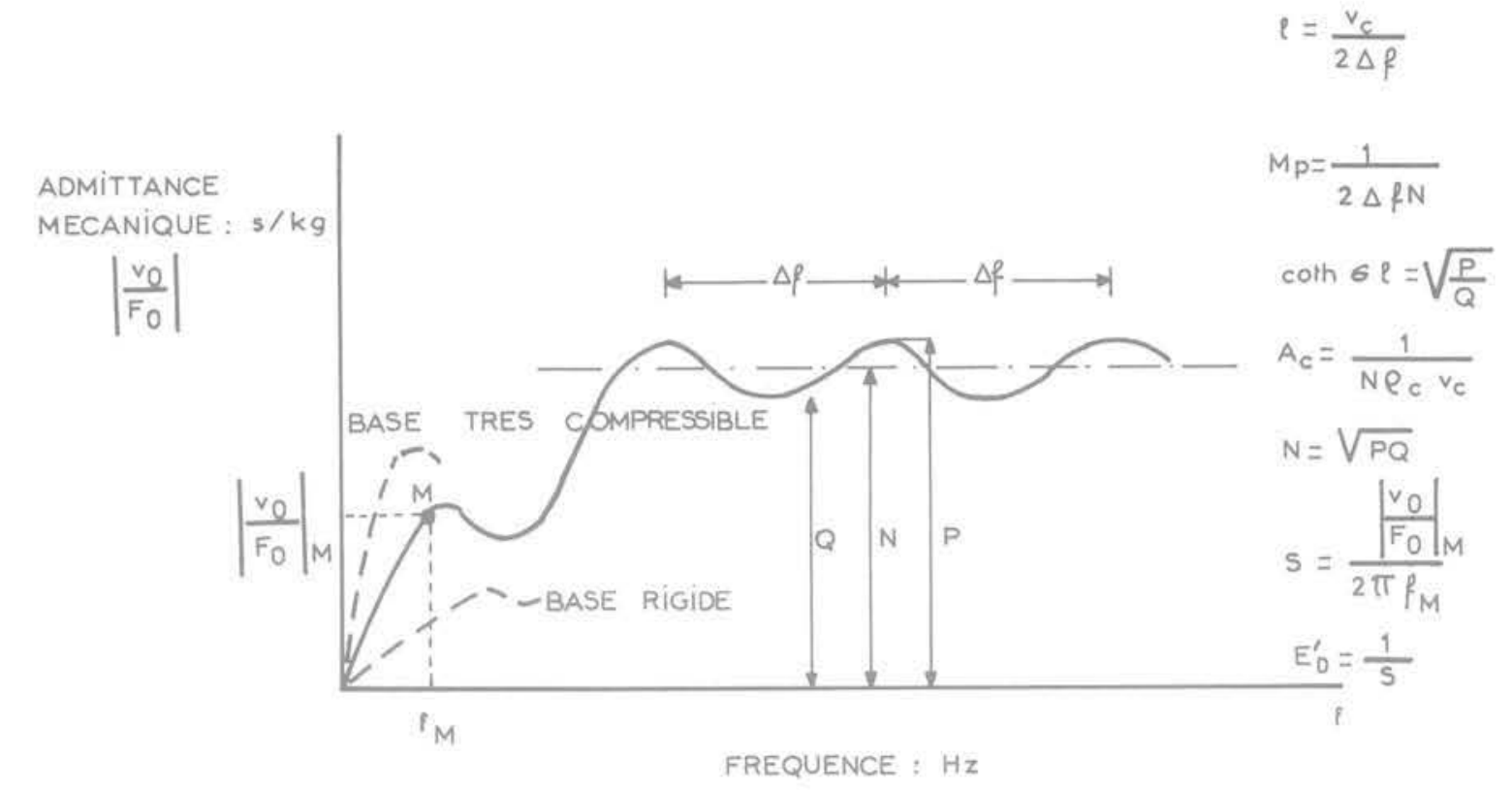

Fig. 2 Courbe de réponse d'un pieu cylindrique

\begin{tabular}{|c|c|c|c|}
\hline $\begin{array}{c}\text { PARAMĖTRE } \\
l_{\max }\end{array}$ & $\begin{array}{c}\text { AMBES } \\
15 \mathrm{~m} \pm 1 \mathrm{~m}\end{array}$ & $\begin{array}{c}\text { FOS-SUR-MER } \\
10 \text { à } 14 \mathrm{~m}\end{array}$ & $\begin{array}{l}\text { PAISLEY } \\
5,5 \text { a } 8 \mathrm{~m}\end{array}$ \\
\hline$A_{C}$ & $\begin{array}{c}0,215-0,898 \mathrm{~m}^{2} \\
\text { circulaire } \\
1,08-1,44 \mathrm{~m}^{2} \\
\text { rectangulaire }\end{array}$ & $\begin{array}{c}0,283-0,786 \mathrm{~m}^{2} \\
\text { circulaire } \\
1,38-3,2 \mathrm{~m}^{2} \\
\text { rectangulaire }\end{array}$ & $\begin{array}{l}0,18 \mathrm{~m}^{2} \\
\text { circulaire }\end{array}$ \\
\hline$l / \mathrm{d}$ & $14 / 1$ à $27 / 1$ & $10 / 1$ à $23 / 1$ & $11 / 1$ à $16 / 1$ \\
\hline$v_{c}$ & $\begin{array}{c}3400-3700 \\
\mathrm{~m} / \mathrm{s}\end{array}$ & $\begin{array}{c}3550-3700 \\
\mathrm{~m} / \mathrm{s}\end{array}$ & $\begin{array}{c}3100-3600 \\
\mathrm{~m} / \mathrm{s}\end{array}$ \\
\hline $\mathrm{v} \mid l$ & $\begin{array}{c}150-350 \\
\mathrm{~m} / \mathrm{s}\end{array}$ & $\begin{array}{c}500-600 \\
\mathrm{~m} / \mathrm{s}\end{array}$ & $\begin{array}{c}\text { pas } \\
\text { mesurée }\end{array}$ \\
\hline$\beta^{\prime}$ & $\sim 150 \mathrm{~m} / \mathrm{s}$ & $\sim 200 \mathrm{~m} / \mathrm{s}$ & $\sim 200 \mathrm{~m} / \mathrm{s}$ \\
\hline $\begin{array}{c}\text { Sol } \\
\text { d'ancrage }\end{array}$ & $\begin{array}{l}\text { Graves } \\
\text { denses }\end{array}$ & $\begin{array}{l}\text { Graves } \\
\text { denses }\end{array}$ & $\begin{array}{l}\text { Moraine } \\
\text { raide }\end{array}$ \\
\hline
\end{tabular}

Tableau 1 Chantier

4 La prévision du tassement des pieux ancrés dans une argile raide et surconsolidée.

L'essai de vibration ne permet pas directement la prévision de la résistance maximale du sol autour d'un pieu. Toutefois, si certaines conditions sont satisfaites, il est possible de prévoir l'ordre de grandeur du tassement d'un pieu sous sa charge de travail à partir de la valeur de sa raideur dynamique $E_{D}^{\prime}$.

Les références 3 et 4 montrent qu'il existe une bonne correspondance entre $E_{D}^{\prime}$ et la raideur statique $E_{S}^{\prime}$ d'un pieu ; cette dernière déterminée à partir de la pente initiale de la courbe "charge - tassement " lors d'un essai de chargement statique. En général, la raideur dynamique est plus forte que la raideur statique, le rapport de $E_{D}^{\prime} / E_{S}^{\prime}$ variant entre 1,0 et 2,0 . De plus, ce même rapport est plus élevé si l'essai dynamique a lieu après l'essai de chargement statique que s'il est effectué auparavant.

Nous avons eu l'occasion d'appliquer la méthode d'impédance à la prévision des tassements des pieux sous un viaduc autoroutier dans la région de Londres. Ces pieux flottants forés, de $1500 \mathrm{~mm}$ de diamètre, traversent entre 3 et $8 \mathrm{~m}$ d'alluvions et de graves de rivière, avant de pénétrer dans l'argile de Londres, qui est une argile raide et surconsolidée, avec le même âge géologique et les mêmes caractéristiques géotechniques que l'argile des Flandres dans le Nord de la France. Tous les pieux sont ancrés au moins de $20 \mathrm{~m}$ dans l'argile, et leurs longueurs varient entre 23 et $37 \mathrm{~m}$. La charge utile demandée pour chaque pieu est de 500 tonnes, à raison de deux pieux par pile de viaduc. 

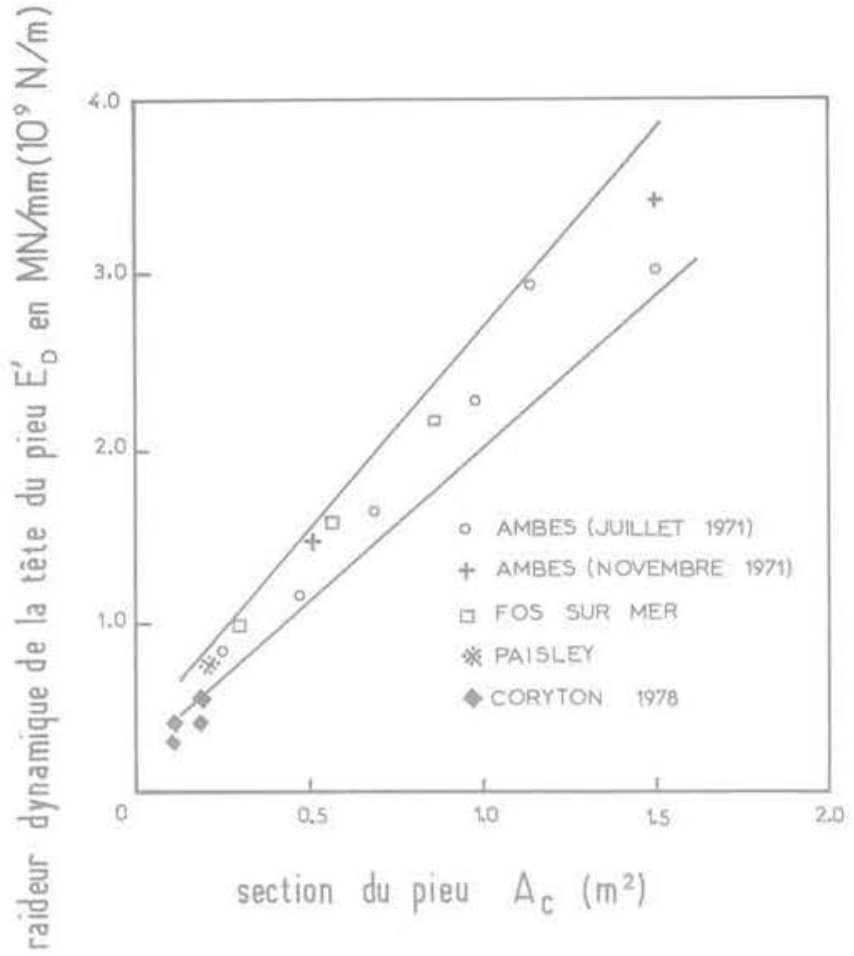

section du pieu $A_{c}\left(m^{2}\right)$

Fig. 3

Etant donné le nombre de pieux relativement faible et la charge élevée, it n'était pas économique de procéder à un essai de chargement statique pour vérifier les hypothèses du projet. Aussi un pieu dessai de $600 \mathrm{~mm}$ de diamètre $a-t-i l$ été construit par le même procédé, et chargé jusqu'à 350 tonnes en tête. La relation charge-tassement obtenue est linéaire jusqu'à 170 tonnes.

Le pieu d'essai a été vibré par le C.E.B.T.P., ainsi que les pieux de $1500 \mathrm{~mm}$. Dans tous les cas, le fond du pieu fut repéré et son intégrité vérifiée.

Le rapport $E_{D}^{\prime} / E_{S}^{\prime}$ mesuré pour le pieu d'essai fut de 1,5 et considéré comme valable pour les pieux de $1500 \mathrm{~mm}$. La raideur dynamique moyenne mesurée pour ces derniers ( $3.78 \mathrm{MN} / \mathrm{mm}$ ou 378 tonnes-force $/ \mathrm{mm}$ ) correspond donc a une raideur statique moyenne de $3,78 / 1,5=2,5$ $\mathrm{MN} / \mathrm{mm}$.

En d'autres termes, une charge de 250 tonnes provoquerait un tassement élastique de $1 \mathrm{~mm}$ pour un pieu de $1500 \mathrm{~mm}$.

BUTLER et MORTON (|5]) ont analysé un nombre important de pieux construits dans l'argile de Londres, et ils montrent que, dans ces cas, la relation charge-tassement d'un pieu est sensiblement linéaire jusqu'à un rapport de charge $P / P_{u}=0,6$ ( $P$ étant la charge appliquée et $P_{u}$ la charge ultime du pieu).

Pour une charge de service de 500 tonnes et une raideur statique de $2,5 \mathrm{MN} / \mathrm{mm}$, le tassement élastique $\dot{\delta}_{e}$ serait de $2,0 \mathrm{~mm}$.

La figure 5 , due à BUTLER et MORTON, indique une valeur de $P / P_{u}=0,3$ pour une valeur correspondante de

$\delta_{e} / d=0,133 \%$.

$\left(\hat{o}_{\mathrm{e}} / \mathrm{d}=\right.$ tassement élastique sur diamètre du pieu).

Ces pieux travaillent donc bien en dessous de la limite élastique du système sol-pieu.

Afin d'estimer le tassement total, la figure 6, due à BUTLER et MORTON, fournit un tassement ó de $2,25 \mathrm{~mm}$ pour $\mathrm{P} / \mathrm{P}_{\mathrm{U}}=0.3$.

5 Détermination de la vitesse des ondes de cisaillement d'un sol par la méthode de l'impédance mécanique.

La connaissance de la vitesse des ondes de cisaillement

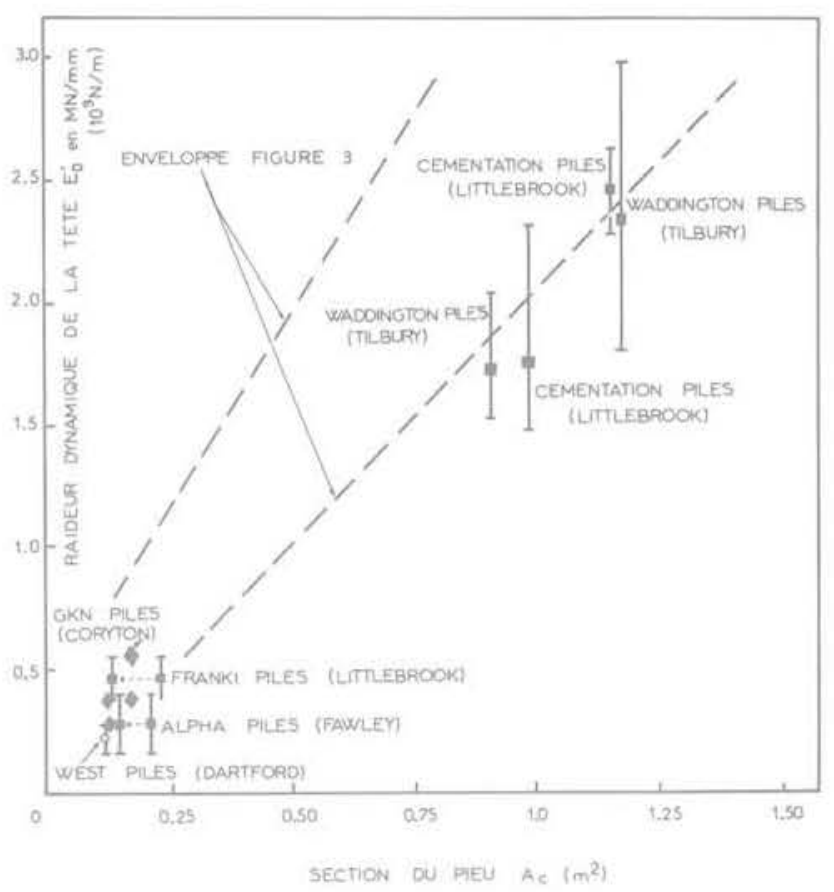

Fig. 4

ou ondes transversales d'un sol est importante puisqu'elle permet l'évaluation de l'ordre de grandeur des paramétres le caractérisant :

$G_{D}=\rho^{\prime} \beta^{\prime 2}=$ module de cisaillement dynamique

et $E_{D}=2(1+v) G_{D}=2(1+v) \rho^{\prime} \beta^{\prime 2}$

$=$ module d'Young dynamique.

La méthode la plus couramment utilisée en Génie Civil est une méthode impulsionnelle (sismique - marteau).

Nous proposons, dans ce paragraphe, de montrer comment l'on peut déterminer, à partir de la réponse d'un cylindre noyé dans un sol et excité verticalement de façon harmonique, la vitesse des ondes transversales de ce sol. Lorsque la réponse à la sollicitation sinusoídale est celle d'un pieu de fondation, comme c'est le cas dans la méthode de l'Impédance Mécanique, la vitesse obtenue tient compte de l'interaction Pieu-Sol et peut être utilisée lors de l'étude de fondations soumises à des sollicitations dynamiques.

\subsection{Evaluation de l'amortissement.}

Le système PIEU-SOL intervenant dans l'amortissement du phénomène vibratoire se réduit au système suivant:

$$
\text { PIEU : } 4 \text { paramètres }\left\{r, L, P_{C}, v_{C}\right)
$$

SOL LATERAL : 2 paramètres ( $\left.p^{\prime}, \beta^{\prime}\right)$

L'amortissement est concrétisé lors de lenregistrement de la courbe en $v_{0} / F_{0}$ par la présence d'un régime de "bosses " qui peuvent être définies avec précision par la lecture des grandeurs $P$ et $Q$ (fig. 1). On démontre (formule 6) que le coefficient d'amortissement du phénomène de propagation est lié aux grandeurs $\mathrm{P}$ et $\mathrm{Q}$ par la formule:

$$
\operatorname{coth}(\sigma l)=\sqrt{\mathrm{P} / \mathrm{Q}}
$$

d'où :

$\sigma=\frac{1}{l} \operatorname{argcoth} \sqrt{\mathrm{P} / \mathrm{Q}}$ 


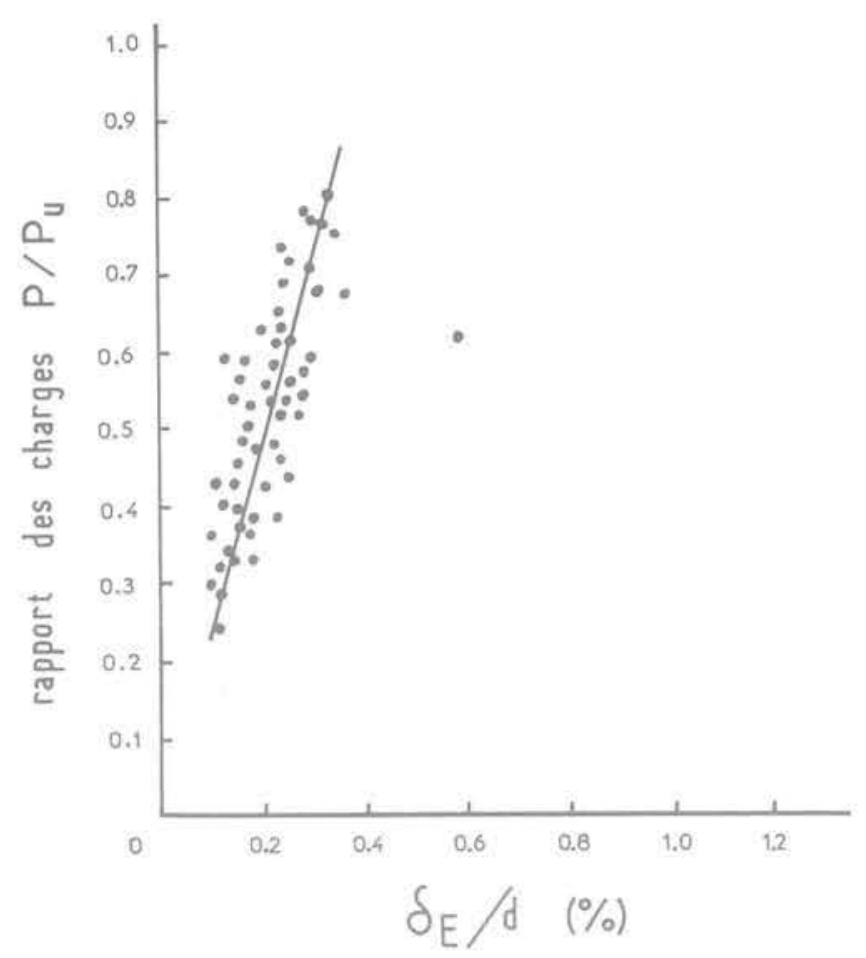

Fig. 5

5.2. Evaluation de la vitesse des ondes de cisaillement du sol.

L'amortissement du phénomène est aussi fonction des paramètres $\left(\rho^{\prime}, \beta^{\prime}\right)$ du sol latéral.

Si l'on se place dans le domaine des hautes fréquences, $\sigma$ peut être évalué avec une bonne précision, à partir de la formule suivante:

$\sigma=\frac{1}{r} \frac{\rho}{\rho_{\mathrm{C}}} \frac{\beta^{\prime}}{v_{\mathrm{C}}}$

d'où :

$\beta^{\prime}=\left(\frac{r \rho_{c} v_{c}}{\rho^{\prime}}\right) \sigma$

ou encore, compte tenu de (8):

$\beta^{\prime}=\left[\frac{r \rho_{c} v_{c}}{\rho^{\prime} t}\right] \operatorname{argcoth}(\sqrt{ } P / Q)$

et

$G_{D}=p^{\prime} \beta^{\prime 2}$

\subsection{Cas d'un terrain à deux couches}

Considérons le cas d'un terrain composé de deux sols dont les caractéristiques sont précisées sur la figure 7.

La vitesse $\beta_{1}^{\circ}$ du sol $n^{\circ} 1$ de surface est déterminée par la formule (2), lorsque l'on prend soin de l'appliquer aux deux premiers écarts de fréquence. On démontre en effet, qu'aux basses fréquences, le sol $n^{\circ} 2$ se comporte comme un ancrage intermédiaire vis-à-vis du sol $n^{\circ} 1$, et que les caractéristiques déduites de l'essai s'appliquent à la première couche. Ces résultats ont été confirmés par des essais de simulation sur odinateur.

II vient : $\beta_{1}^{\prime}=\frac{r \rho_{c} v_{c}}{\rho_{1}^{\prime} l_{1}} \quad \operatorname{argcoth}\left(\sqrt{P_{1} / Q_{1}}\right)$

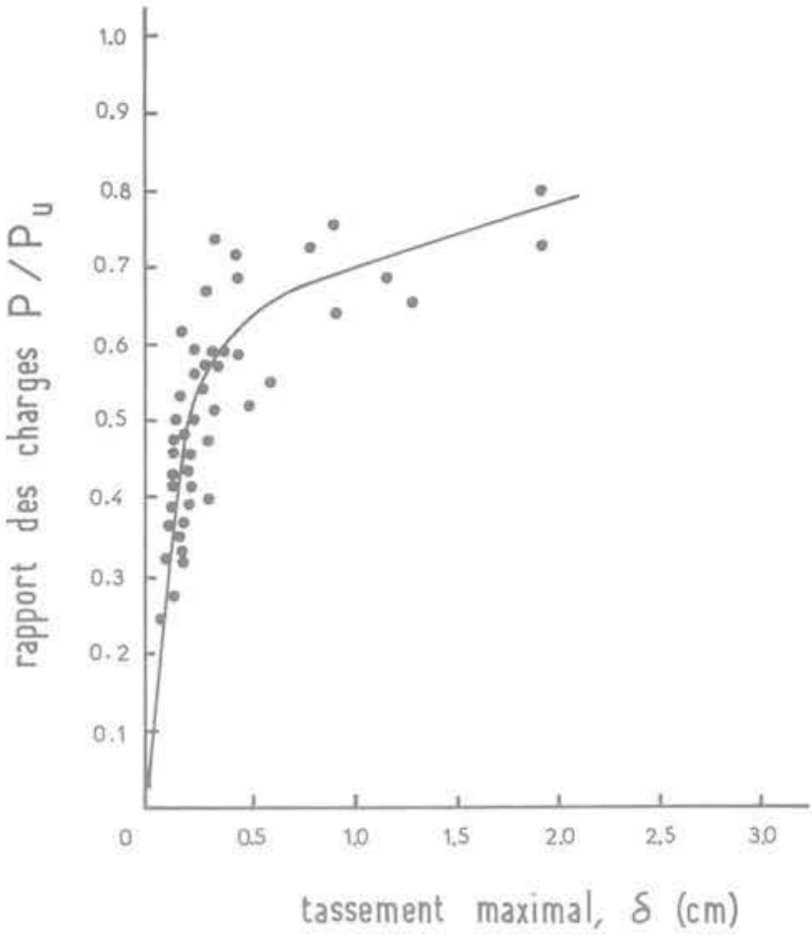

Fig. 6

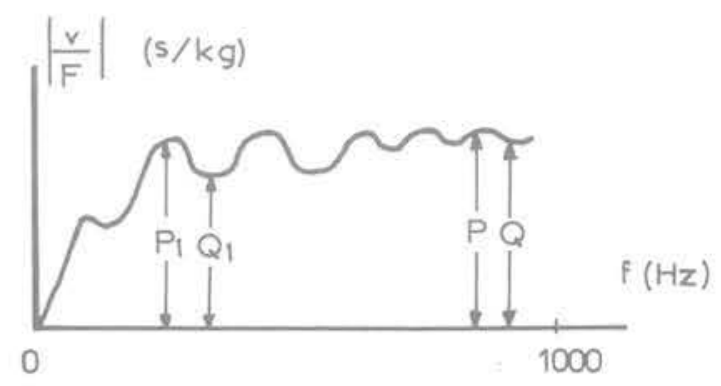

Fig. 7 
Aux fréquences relativement élevées $(f \approx 1000 \mathrm{~Hz}$ ), l'amortissement obtenu $\sigma l$ tient compte des caractéristiques des deux couches. II est déterminé par:

$\sigma l=\sigma_{1} l_{1}+\sigma_{2} l_{2}$

D'autre part : $\sigma l=\operatorname{argcoth}(\sqrt{\mathrm{P} / \mathrm{Q})}$

d'où : $\sigma_{2} l_{2}=\sigma l-\sigma_{1} l_{1}=\frac{l_{2}}{\mathrm{r}} \frac{\rho_{2}^{\prime}}{\rho_{\mathrm{C}}} \frac{\beta_{\mathrm{C}}^{\prime}}{\mathrm{v}_{2}}$

La vitesse $\beta_{2}^{\prime}$ du sol $n^{\circ} 2$ est alors donnée par la formule : $\beta_{2}^{\prime}=\frac{r \rho_{C} v_{C}}{\rho_{2}^{\prime} l_{2}}\left[\operatorname{argcoth}(\sqrt{P / Q})-\operatorname{argcoth}\left(\sqrt{P_{11} / Q_{1}}\right)\right]$

Dans la pratique, pour un bon béton, on pourra utiliser les formules simplifiées suivantes:

$\rho_{\mathrm{C}}=2400 \mathrm{~kg} / \mathrm{m}^{3} \mathrm{v}_{\mathrm{C}}=4000 \mathrm{~m} / \mathrm{s}$

$\rho^{\prime}=1800 \mathrm{~kg} / \mathrm{m}^{3}$

a) Cas d'un monocouche

$\beta^{*}=5,3 \cdot 10^{3} \frac{r}{l} \operatorname{argcoth}(\sqrt{P / Q})$

b) Cas d'un bicouche

$\beta_{1}^{\prime}=5,3 \cdot 10^{3} \frac{r}{l_{1}} \operatorname{argcoth}\left(\sqrt{P_{1} / Q_{1}}\right)$

$(\mathrm{m} / \mathrm{s})$

$(\mathrm{m} / \mathrm{s})$

$\beta_{2}^{\prime}=5,3 \cdot 10^{3} \frac{r}{l_{2} i}\left\lfloor\operatorname{argcoth}(\sqrt{P / Q})-\operatorname{argcoth}\left(\sqrt{P_{1} / Q_{1}}\right\rfloor\right.$

\subsection{Résultats d'essais}

Le tableau 2 regroupe les différentes valeurs de la vitesse des ondes de cisaillement obtenues à partir de la vibration harmonique de pieux cylindriques en béton pour différents types de sol.

\section{L'interaction PIEU-SOL déduite de la partie réso-} nante de la courbe de réponse

Nous avons vu dans le paragraphe 2 que l'amortissement de la courbe de réponse dans la partie résonante est représenté par le terme $\sigma l$, et que coth $(\sigma l)=\sqrt{(\mathrm{P} / \mathrm{Q})}$.

BRIARD a montré qu'il est possible de déterminer les raideurs limites du système pieu-sol pour les deux cas extrêmes :

1/ pieu sur base infiniment rigide, avec une raideur maximale, $E_{\text {max }}^{\prime}$ donnée par:

$E_{\max }^{\prime}=E_{\infty}^{\prime} \operatorname{coth}(\sigma l)=E_{\infty}^{\prime} \sqrt{P / Q}$

2/ pieu infiniment souple, se comportant comme un pieu entièrement coupé à sa base, avec une raideur minimale.

$E_{\min }^{\prime}=E_{\infty}^{\prime}$ th $(\sigma l)=E_{\infty}^{\prime} \sqrt{Q / P}$

$E_{\infty}^{\prime}$ est la raideur d'un pieu flottant d'une longueur infinie fondé entièrement dans le sol latéral, et peut être exprimée par:

$E_{\infty}^{\prime}=A_{C} \cdot E_{c} \cdot \sigma$

II est intéressant de comparer la raideur mesurée à basse fréquence, $E_{D}^{\prime}$ avec ces valeurs limites. A titre d'exemple.

\begin{tabular}{l|c}
\hline \multicolumn{1}{c|}{ Nature du Sol } & Vitesse (m/s) \\
\hline Vase - Tourbe & $110-150$ \\
\hline $\begin{array}{l}\text { Argile molle } \\
\text { Sable lâche }\end{array}$ & $140-200$ \\
\hline $\begin{array}{l}\text { Argile normalement } \\
\text { consolidée } \\
\text { Sable peu compact }\end{array}$ & $200-250$ \\
\hline $\begin{array}{l}\text { Argile légèrement } \\
\text { surconsolidée }\end{array}$ & \\
$\begin{array}{l}\text { Sable moyennement } \\
\text { compact }\end{array}$ & \\
Calcaire fissuré & \\
Craie altérée & \\
\hline
\end{tabular}

Tableau 2

\begin{tabular}{|c|c|c|c|c|c|}
\hline \multirow{2}{*}{\multicolumn{2}{|c|}{ PIEU }} & \multicolumn{4}{|c|}{ RAIDEUR (MN/mm) } \\
\hline & & $E_{D}^{\prime}$ & $\mathrm{E}_{\min }^{\prime}$ & $E_{\infty}^{\prime}$ & $E_{\text {max }}^{\prime}$ \\
\hline 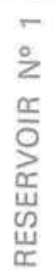 & $\begin{array}{l}1 \\
2 \\
3 \\
4 \\
5 \\
6 \\
7\end{array}$ & $\begin{array}{l}0,417 \\
0,333 \\
0,625 \\
0,278 \\
0,294 \\
0,263 \\
0,294\end{array}$ & $\begin{array}{l}0,385 \\
0,175 \\
0,333 \\
0,167 \\
0,172 \\
0,182 \\
0,145\end{array}$ & $\begin{array}{l}0,500 \\
0,294 \\
0,476 \\
0,286 \\
0,303 \\
0,303 \\
0,270\end{array}$ & $\begin{array}{l}0,625 \\
0,500 \\
0,667 \\
0,500 \\
0,526 \\
0,500 \\
0,500\end{array}$ \\
\hline $\begin{array}{l}N \\
0 \\
z \\
\frac{\pi}{0}\end{array}$ & $\begin{array}{r}8 \\
9 \\
10 \\
11\end{array}$ & $\begin{array}{l}0,167 \\
0,278 \\
0,294 \\
0,192\end{array}$ & $\begin{array}{l}0,063 \\
0,069 \\
0,067 \\
0,067\end{array}$ & $\begin{array}{l}0,152 \\
0,159 \\
0,167 \\
0,167\end{array}$ & $\begin{array}{l}0,370 \\
0,370 \\
0,417 \\
0,417\end{array}$ \\
\hline$\underset{\underset{\sim}{\dddot{x}}}{\underset{\sim}{w}}$ & $\begin{array}{c}12 \\
\text { (cassé) }\end{array}$ & 0,100 & 0,093 & 0,250 & 0,685 \\
\hline
\end{tabular}

Tableau 3

nous présentons ici les résultats obtenus pour un chantier à Fawley, Angleterre. II s'agit de deux réservoirs pétroliers de $80 \mathrm{~m}$ de diamètre chacun, reposant sur des radiers minces en béton armé fondés sur pieux battus moulés, de type Alpha.

Ces pieux à base élargie, de $420 \mathrm{~mm}$ de diamètre et d'environ $10 \mathrm{~m}$ de long, traversaient une couche de vase et de tourbe pour se fonder dans une couche, de graves d'estuaire de qualité et d'épaisseur variables.

Au moment de l'essai de chargement préliminaire du premier réservoir, des désordres au niveau du bord du radier ont été constatés. Lorsque la cuve métallique fut enlevée, on a constaté des ondulations dans le radier avec des tassements différentiels allant jusqu'à $60 \mathrm{~cm}$. L'essai de chargement préliminaire du deuxième réservoir fut arrêté à une charge équivalente à $20 \%$ de la charge de service. 
Le C.E.B.T.P. fut appelé à contrôler l'intégrité de certains de ces pieux, surtout dans les zones d'instabilité. Parmi les 43 pieux auscultés, il y avait 11 pieux entiers et sans défaut. Nous avons présenté dans le tableau 3 les résultats de $E_{p}^{\prime}, E_{\min }^{\prime}, E_{\infty}^{\prime}$ et $E_{\max }^{\prime}$ pour ces 11 pieux, ainsi que pour un douzième pieu qui était cassé à $7 \mathrm{~m}$ de profondeur dans la vase.

Les valeurs de $E^{\prime}$ min montrent que le sol latéral sous le réservoir $n^{\circ} 1$ est nettement plus raide que celui sous le réservoir $n^{\circ} 2$. Cette observation est confirmée par les valeurs de E'co.

Les valeurs de $E_{D}^{\prime}$ mesurées se situent autour de $E_{\infty}^{\prime}$ pour le premier réservoir, tandis qu'elle tombent entre $E_{\infty}^{\prime}$ et $E_{\text {max }}^{\prime}$ pour le réservoir $n^{\circ} 2$. Ces résultats indiquent que ces pieux travailient bien en pointe, et non au frottement latéral.

\section{Conclusions}

Lorsque le C.E.B.T.P. a développé la méthode d'auscultation par impédance mécanique, il y a dix ans, la limite supérieure de l'élancement d'un pieu, au-delà de laquelle l'amortissement devenait trop important, était considérée comme étant de l'ordre de 10 . De plus, on ne considérait, a l'époque, que l'intégrité du pieu, sans prendre en compte les renseignements concernant l'interaction du pieu avec le sol de fondation.
Or il a été démontré, récemment, que cette méthode permet d'ausculter des pieux possédant un élancement allant jusqu'à 50 , lorsque le sol latéral est peu raide, et qu'il est possible d'interpréter les courbes de réponse en fonction de la nature du sol et de son interaction avec le pieu. L'existence d'une corrélation entre la raideur dynamique mesurée par cette méthode, et la raideur déduite d'un essai de chargement statique permet un choix rationnel des pieux à vérifier par chargement, et donc une économie importante lors d'une opération de construction.

\section{Références bibliographiques}

1 - PAQUET J. «Etude vibratoire des pieux en béton: réponse harmonique et impulsionnelle; application au contrôle \& Annales ITBTP - série EM/111 (mai 1968).

2 - PAQUET J. et BRIARD M. « Contrôle non destructif des pieux en béton ». Annales ITBTP - série EM/128 (mars 1975).

3 - DAVIS A.G. et DUNN C.S. "from theory to field experience with the non-destructive vibration testing of piles \#. Proc. Instn. Civ. Engrs. Part 2 (1974), 57, pp. 571-593.

4 - DAVIS A.G. et ROBERTSON S.A. " Vibration Testing of Piles 》. Structural Engineer (juin 1976).

5 - BUTLER F.G. et MORTON K. a Specification and Performance of test piles in London Clay 》. Proc. Symp. I.C.E. on Behaviour of Piles (1970), pp. 17-26. 
